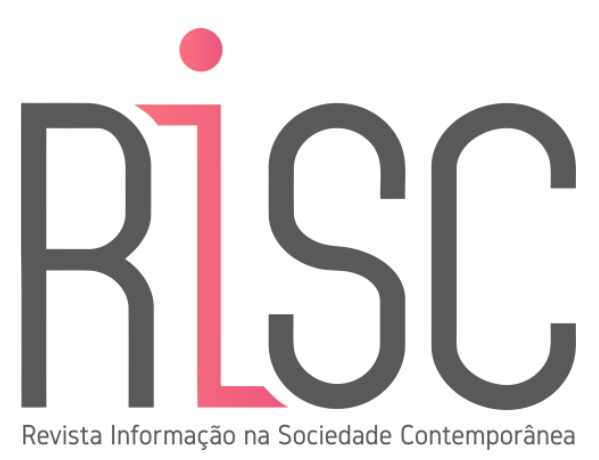

\title{
A INFLUÊNCIA DA INTELIGÊNCIA DE MERCADO NO PLANEJAMENTO DE UMA CIDADE INTELIGENTE
}

\author{
André Luiz Gomes* \\ Mestrando em Informação e Gestão de Organizacional \\ https://orcid.org/0000-0002-6090-5480 E-mail: anlgomes@hotmail.com \\ Taiane Ritta Coelho* \\ Doutora em Administração \\ https://orcid.org/0000-0003-2607-0704 E-mail: taianercoelho@gmail.com \\ * Universidade Federal do Paraná, Programa de Pós-Graduação em Gestão da Informação, \\ Departamento de Ciência e Gestão da Informação, Curitiba, PR, Brasil.
}

Submetido em: 25-11-2020 Reapresentado em: 10-02-2021 Aceito em: 21-02-2021

\section{RESUMO}

Com o surgimento de novas cidades inteligentes empresas evoluem seus processos, produtos e posicionamento de mercado com o intuito de aumentar vendas ou buscar parcerias que propiciem o desenvolvimento de seus negócios, porém quais serão as características desse novo mercado? Qual o perfil do novo consumidor? Será que prefeituras estão preparadas para auxiliar empresas a captarem a demanda gerada no processo de construção de uma cidade inteligente? Visando responder esses questionamentos conduzimos essa pesquisa exploratória com auxílio de dados abertos para nos mostrar quais as contribuições ou oportunidades que a inteligência de mercado poderá proporcionar ao cenário empreendedor de uma cidade inteligente. 
Palavras-chave: Smart City. Empreendedorismo. Cidades Inteligentes. Marketing Territorial. Inteligência de Mercado, inteligência competitiva.

\section{THE INFLUENCE OF MARKET INTELLIGENCE IN SMART CITIES PLANNING}

\section{ABSTRACT}

As the emergence of new smart companies evolves their processes, products and market positioning as an idea to increase sales or seek partnerships that promote or develop their business, why would these be the characteristics of this new market? What is the profile of the new consumer? Are city halls prepared to help companies capture on demand generated by the process of building a smart city? In order to answer these questions, we conducted this exploratory research with the help of open data to show us what contributions or opportunities market intelligence can provide to the entrepreneurial community of a smart city.

Keywords: Smart City. Entrepreneurs. Marketing. Marketing Intelligence.

\section{INTRODUÇÃO}

Lazaroiu e Roscia (2012) acredita que o futuro das cidades inteligentes será muito desafiador, pois surge a necessidade de novos modelos de cidades onde a tecnologia estará a serviço das pessoas melhorando o seu cenário econômico junto com o aumento no nível da qualidade de vida social. A busca intensa pela melhoria contínua nos direciona cada vez mais à necessidade de desenvolvermos tecnologias, métodos, processos ou sistemas que auxiliem na realização das atividades, consumindo o mínimo de recursos possíveis.

A gestão da informação (GI) proporcionou para as empresas e a sociedade acesso a um nível de aprimoramento tecnológico que, por sua vez, contribuiu para um aumento na velocidade de resposta aos estímulos internos e externos do ambiente em que atuam (SHAW et al., 2001). Esses estímulos caracterizam-se por tendências sociais, interações com mercados emergentes, questões de sustentabilidade, saúde, bem-estar e educação. Em outras palavras, significa que a GI contribui para que processos possam ir além das empresas influenciando o mercado direta e indiretamente (SHAW et al., 2001). 
Conforme defendido por Lazaroiu e Roscia (2012), o futuro das cidades inteligentes torna-se muito desafiador, pois surge a necessidade de novos modelos de cidades onde a tecnologia estará a serviço das pessoas melhorando o seu cenário econômico junto com o aumento no nível da qualidade de vida social. Essas necessidades geradas por uma cidade que atinge um nível considerável de processos inteligentes geralmente movimenta a economia, desenvolvendo stakeholders regionais (comércio, pessoal, governos). Mesmo que essas necessidades funcionem como motor para o desenvolvimento, tornam-se necessárias ações direcionadas de modo que seja possível otimizar o desenvolvimento informacional de uma cidade, para tal necessidade pode-se utilizar metodologias de inteligência de mercado visando capitalizar essas oportunidades e potencializar o cenário empreendedor de uma cidade inteligente.

Existem estudos que divergem em relação aos conceitos dos termos de inteligência de mercado ou inteligência competitiva. De acordo com Weng (2020), a inteligência de mercado é a habilidade sistêmica de coletar ou estratificar informações que suportam decisões mercadológicas. Ou seja, a inteligência de mercado é um componente primordial para as atividades mercadológicas, sendo de grande relevância para manter uma empresa sustentável proporcionando aprendizagem a respeito de comportamento de clientes, concorrentes, parceiros ou mercados internacionais em cada tipo de operações. Já a inteligência competitiva é uma junção de informações de origem tecnológicas, ambientais, sociais, financeiras, mercadológicas ou de produtos que necessitam de uma síntese teórica no tratamento da informação auxiliando na tomada de decisão para as organizações de forma estratégica e organizada (TARAPANOFF, 2006). Conforme Dalmarco (2020) e Teixeira (2011), devido à proximidade das definições entre os termos inteligência de mercado e inteligência competitiva, neste estudo iremos adotar um conceito único para ambas que considera a inteligência de mercado ou inteligência competitiva como partes da inteligência empresarial e engloba principalmente informações sobre mercado e concorrentes.

Diante desse cenário, endente-se que os usos das ferramentas de inteligência de mercado com a gestão da informação podem influenciar diretamente no 
planejamento de uma cidade inteligente, agregando valor econômico ao mercado empresarial ou social. Desta forma, a pesquisa buscará identificar qual influência da inteligência de mercado no planejamento de uma cidade inteligente, potencializando o desenvolvimento empresarial de um território. Serão investigadas as três principais cidades inteligentes brasileiras de acordo com o estudo produzido pela consultoria Urban Systems (2020), Campinas - SP (como referência tecnológica), São Paulo - SP (como referência em mobilidade e inovação) e Curitiba-PR (como referência em urbanismo e capital da fintech, startups especializados em transações financeiras on line), contribuindo direta e indiretamente para o entendimento do complexo ecossistema que envolvem as cidades inteligentes.

\section{FUNDAMENTAÇÃO TEÓRICA}

A fundamentação teórica deste projeto engloba os seguintes tópicos: cidades inteligentes; inteligência de mercado; e marketing de território. Estes tópicos serão apresentados a seguir, bem como a relação entre eles.

\subsection{CIDADES INTELIGENTES E SEUS DESAFIOS}

De acordo com Giffinger et al. (2007) uma cidade inteligente caracteriza-se por um bom desempenho econômico, de pessoas, mobilidades e meio ambiente combinado com doações inteligentes de cidadãos independentes. Para Kourtit, Nijkamp e Stough (2019), uma cidade inteligente caracteriza-se por uma interação do conhecimento, capital humano, capital de infraestrutura, capital social e capital empresarial unificados em uma estratégia criativa que visa melhorar o desempenho socioeconômico, ecológico, logístico e competitivo das cidades.

Para Carvalho e Maia (2016), a necessidade de desenvolvimento em uma cidade leva a questionamentos de como um governo deveria planejar melhor essas ações visando potencializar a produtividade dos setores de base social, bem como saúde, educação, segurança e economia. Em complemento, Cidrais (2001) enfatiza que 
marketing territorial potencializa a competitividade entre empresas locais forçando o desenvolvimento territorial, descentralizando o poder processual gerando novas oportunidades de desenvolvimento. Partindo dessas referencias, entende-se que um mapeamento adequado de cenários sociais proporcionará um maior aproveitamento dessas iniciativas de forma coerente com as dificuldades de cada cidade, buscando o desenvolvimento igualitário em grande parte do território através dessa competitividade, obtendo maior eficácia no processo de cidades inteligentes focando no desenvolvimento empresarial.

Castells (2005) defende que economias em desenvolvimento se articulam entre si de acordo com o fluxo dinâmico da economia global, aumentando taxas de crescimento, produtividade e industrialização de uma sociedade. Esse conceito é conhecido como sociedade em rede e é apoiado em três processos: geração e difusão de novas tecnologias, transformação do trabalho com crescimento altamente qualificado, autônomo capaz de inovar e de adaptar-se a mudanças globais, difusão de uma nova organização em torno de redes. Somente quando uma organização atinge essas três condições é possível sustentar a competitividade a longo prazo.

De acordo com a perspectiva de De Jong et al. (2015), as cidades do mundo moderno estão se desenvolvendo cada vez mais consumindo recursos naturais e devido a esse fato é necessário examinar as ramificações dessa escassez de fontes energéticas não renováveis e, consequentemente, proteger heranças naturais e fontes de energias renováveis tornou-se um ponto importante na manutenção de sustentabilidade de uma cidade inteligente.

\subsection{O CENÁRIO EMPREENDEDOR EM CIDADES INTELIGENTES}

Em pesquisa produzida para o Serviço de Apoio às Micro e Pequenas Empresas (SEBRAE), a posição mundial do Brasil em relação aos aspectos de conectividade e a quantidade de pessoas vivendo em áreas urbanas colocam lado a lado a revolução digital e a urbanização massiva originando cidades hiper conectadas e colaborativas, onde o indivíduo cria formas rápidas e inteligentes de interagir entre si. Nesse sentido, 
as cidades inteligentes são uma resposta a necessidade de adaptação ao ambiente urbano, diferenciando das cidades tradicionais pelo uso das Tecnologias da Informação e da Comunicação (TIC). Esse ambiente pode ser extremamente favorável aos pequenos negócios, inserindo-os em uma nova cadeia de produção e fornecimento.

De acordo como o Ranking Connected Smart Cities produzido pela consultoria Urban Systems que tem como objetivo mapear as cidades com maior potencial de desenvolvimento no Brasil através de indicadores que qual ficam as cidades mais inteligentes e conectadas do país, publicado também pelo site BrasilLab uma hub de inovação que acelera soluções e conecta empreendedores com o Poder Público, podemos observar a importância de ações empreendedoras para as cidades inteligentes, pois serão elas responsáveis por suprir as necessidade de novas demandas à medida que as cidades se desenvolvem.

Ranking Connected Smart Cities é composto de 11 indicadores elaborados através de pesquisas de campo, revisões literárias e entrevistas com especialistas em cada eixo de impacto, sendo mobilidade e acessibilidade, urbanismo, saúde, educação, energia, economia, meio ambiente, tecnologia e inovação, segurança, empreendedorismo, governança. Como resultado desse estudo, o ano 2019 apresentou as cidades de Campinas - SP seguida por São Paulo - SP e em terceiro lugar Curitiba - PR. Todas destacando-se no quesito de desenvolvimento tecnológico e empreendedorismo.

Analisando os dados de performance econômica para as três primeiras cidades inteligentes segundo a consultoria Urban System, podemos perceber que o setor empresarial que possui maior representatividade no produto interno bruto (PIB) no conjunto das três cidades é o setor de comercio e serviços representando $64 \%$ de todo o PIB arrecadado das mesmas, em comparativo com os números do Brasil temos o percentual de $56 \%$ no mesmo setor, apresentando uma diferença de oito pontos percentuais acima do resultado nacional. De acordo com a mesma fonte de informação, estima-se que $80 \%$ das empresas que compõem o indicador de total de empresas do setor de comércio e serviço são micro e pequenas empresas. 
Fazendo um prévio cruzamento entre o resultado do ranking de Urban System com os dados fornecido pelo portal Data SEBRAE, podemos visualizar a influência do processo das tecnologias da informação e comunicação (TIC) das cidades inteligentes no cenário empreendedor, atuando em conjunto com as demais frentes potencializando o capital estratégico de cada cidade.

Municípios associados a grandes empresas observam nas cidades inteligentes uma fonte de diversificação de receitas com empresas de aplicações derivadas das mesmas áreas, por exemplo empresas de venda de software proprietário, centro de operações, serviços de consultorias e produção de dados fechados. Podemos citar a parceria entre governo do estado do Rio de Janeiro com a IBM na construção do centro de operações para a prefeitura do Rio de Janeiro, possibilitando em tempo real o monitoramento de tráfego, transporte público, abastecimento de água entre outros serviços de utilidade pública. O sistema adaptou-se de maneira tão satisfatória que a IBM desenvolveu módulos de mapeamento para a comercialização em outras cidades que tenham interesse na automatização de seus processos Kitchin (2014).

Kourtit, Nijkamp e Stough (2019) defendem a ideia de que as melhores oportunidades para iniciativas empreendedoras são oferecidas por regiões altamente funcionais que combinem um grande mercado consumidor e uma alta acessibilidade a mercados vizinhos. Geralmente essas regiões possuem como principal característica uma renda per capita expressiva. Os empreendedores que conseguem estruturar suas empresas dentro dessas regiões possuem a vantagem competitiva da concentração de um grande potencial de consumidores com um alto poder de compras se comparados com outras empresas que estão a margem dessas regiões. Em outras palavras, regiões com uma maior acessibilidade e mobilidade urbana contribuem diretamente para o desenvolvimento empresarial de uma cidade, com tudo é papel dos governantes criarem fomentos necessários para que esse ecossistema se amplie.

Entendendo a complexidade e a oportunidade que o cenário empreendedor traz a uma cidade, governos iniciam uma corrida do ouro com iniciativas que contribuem e dão suporte a esse nicho tecnológico. Por exemplo, Campinas concentra grande parte do polo tecnológico nacional, abrigando empresas como IBM, Dell, 
LeNovo e HP entre outras. A cidade também possui doze centros de pesquisas e desenvolvimento como a Companhia de Desenvolvimento do Polo de Alta Tecnologia (CIATEC), o Centro de Pesquisa e Desenvolvimento em Telecomunicações (CPQD), o Parque Científico e Tecnológico da Unicamp e o Techno Park um empreendimento que reúne 66 empresas, sendo 43 do setor de tecnologia. Já Curitiba a capital paranaense traz, entre outras ações, o Vale do Pinhão, uma iniciativa com propósito de fortalecer e potencializar o ambiente de inovação por meio do empreendedorismo, economia criativa e tecnologia alinhado aos Objetivos de Desenvolvimento Sustentável (ODS) da Organização das Nações Unidas (ONU) envolvendo secretarias municipais e o ecossistema de inovação de Curitiba, que é composto por universidades, aceleradoras, incubadoras, fundos de investimento, centros de pesquisa e desenvolvimento, startups, movimentos culturais e criativos. Para São Paulo podemos citar duas iniciativas focadas em auxiliar o desenvolvimento estruturado da cidade potencializando o ecossistema empreendedor e de inovação, são eles; 011Lab espaço dentro da Prefeitura de São Paulo focado em inovação no setor público, buscando unir pessoas de dentro e fora da administração municipal para desenvolvimento de soluções inovadoras para problemas de interesse público, impactando na gestão e melhorando os serviços aos cidadãos. Outra iniciativa da prefeitura da São Paulo é o MobiLab+, uma organização reconhecida nacional e internacionalmente pela inovação no segmento de mobilidade urbana, cuja abertura de dados permitiu o lançamento de uma série de aplicativos e ferramentas com foco no transporte e no trânsito.

Essa movimentação em prol do desenvolvimento empreendedor torna-se cada vez mais intensa à medida que a sinergia entre os pilares estratégicos de uma cidade inteligente aumenta, gerando maior qualidade de vida aos cidadãos. Sendo no quesito mobilidade urbana com formas menos poluentes, rápidas e econômicas ou no quesito de acessibilidade proporcionando ao usuário maiores comodidades, segurança e conforto, consumindo cada vez mais produtos ou serviços com o intuito de suprir sensações de escassez em relação a tempo, dinheiro, comida, laser, educação, saúde, moradia entre outros. 


\subsection{INTELIGÊNCIA DE MERCADO E INTELIGÊNCIA COMPETITIVA}

Em sua tese de doutorado, Trombin (2014), faz um relato histórico do uso de dados e informações utilizadas por empresas desde 1930 e nesta tese traz a evolução e a utilização do conceito de inteligência de mercado ou inteligência competitiva. Em grandes linhas, podemos resumir que inteligência competitiva se baseia em dados sistemáticos, estruturados voltados ao mapeamento de concorrentes, como estratégias de vendas, participação de mercado, aquisições ou lançamento de novos produtos. Para inteligência de mercado, temos uma análise baseada em insights, buscando mapear singelas alterações no mercado, criando cenários de simulações sem predefinição de dados gerando alertas de qualquer ameaça que possa comprometer a estratégia da empresa. O quadro 1 apresenta uma síntese desses dois conceitos.

Quadro 1 - Conceito de Inteligência de Mercado e Inteligência Competitiva

\begin{tabular}{|c|c|c|}
\hline Termo & Definição do termo & Autor (es) \\
\hline \multirow[t]{5}{*}{$\begin{array}{l}\text { Inteligência } \\
\text { competitiva }\end{array}$} & $\begin{array}{l}\text { Programa sistemático para levantamento e análise da informação } \\
\text { sobre as atividades dos concorrentes e das tendências de negócio no } \\
\text { geral, de forma a promover as metas da organização. }\end{array}$ & Kahaner (1997) \\
\hline & $\begin{array}{l}\text { Processo legal e ético de coletar, analisar e aplicar informações sobre } \\
\text { as potencialidades, as vulnerabilidades e as intenções da } \\
\text { concorrência. }\end{array}$ & $\begin{array}{l}\text { Prescott e Miller } \\
(2002)\end{array}$ \\
\hline & $\begin{array}{l}\text { Processo de levantar informações acerca das condições competitivas } \\
\text { e dos riscos (incluindo a ameaça de novos entrantes, produtos } \\
\text { substitutos, concorrentes e fornecedores), para que esse } \\
\text { conhecimento seja aplicado na tomada de decisão. }\end{array}$ & Fuld (2006) \\
\hline & $\begin{array}{l}\text { Processo sistemático, com o esforço direcionado para coletar } \\
\text { informações de forma ética, sintetizar, analisar a concorrência, } \\
\text { mercados e ambiente externo a fim de produzir insights para a } \\
\text { tomada de decisão. }\end{array}$ & Fleisher (2008) \\
\hline & $\begin{array}{l}\text { Processo pelo qual as organizações reúnem informações acionáveis } \\
\text { sobre competidores e o ambiente competitivo e idealmente aplicam } \\
\text { essas informações em seus processos de planejamento e tomada de } \\
\text { decisão, no intuito de melhorar o desempenho da empresa. }\end{array}$ & $\begin{array}{l}\text { Fleisher e } \\
\text { Bensoussan (2009) }\end{array}$ \\
\hline $\begin{array}{l}\text { Inteligência de } \\
\text { mercado }\end{array}$ & $\begin{array}{l}\text { Processo que transforma informações em insights que ajudam a } \\
\text { organização a entender o ambiente de negócios e a tomar decisões } \\
\text { para competir e crescer no mercado em que atua. Sâo informações } \\
\text { sobre os atores do mercado e os tópicos estrategicamente relevantes. } \\
\text { Envolve a realização de projetos ad hoc, relacionados com decisões } \\
\text { muito especificas, e de monitoramento continuo do mercado, para } \\
\text { manter a organização alerta sobre os acontecimentos do mercado, } \\
\text { das oportunidades e das ameaças. }\end{array}$ & $\begin{array}{l}\text { Hedin, Hirvensalo e } \\
\text { Vaarnas (2011) }\end{array}$ \\
\hline
\end{tabular}

Fonte: Trombin (2014) 
Neste projeto, o foco de investigação será a inteligência de mercado. Os principais objetivos da inteligência de mercado são aumentar a capacidade competitiva das empresas; prever de maneira confiável a evolução do ambiente competitivo, das ações do concorrente, das necessidades dos clientes, dos resultados de mudanças políticas, prover apoio ao processo de tomada de decisão estratégica, identificar ameaças e oportunidades por meio da interpretação de sinais fracos e alertas antecipados, processar dados para produzir conhecimento sobre concorrentes, reduzir o tempo de reação e resposta e identificar estratégias de mercado, reduzir custos, mitigar riscos e aumentar produtividade.

Martinet (2010) defende que o monitoramento de concorrentes de forma rápida e efetiva, para isso é necessário que o sistema de informação seja estruturado de maneira de possibilite antecipar suas ações, introduzir novos produtos e entrar em novos mercados. Entendendo que a captura e uso de forma estratégica de sinais fracos do mercado evitaria surpresas em razão de rupturas de mercado ou descontinuidades de produtos.

\subsection{A RELAÇÃO ENTRE INTELIGÊNCIA DE MERCADO, MARKETING TERRITORIAL E CIDADES INTELIGENTES}

Com o intuito de desenhar uma estratégia que englobe essa complexidade de cenários e pluralização de informações foi criado em 1993 por Kotler, Haider e Rein o conceito de marketing territorial (MT), que apresenta uma abordagem estratégica ao marketing de lugares, comparando cidades a um produto, abordando a importância dos clusters como forma de diferenciação competitiva e da necessidade de funcionamento em redes focadas em reforçar ligações e criar condições para a competitividade de um país (ARAGONEZ; ALVES, 2013, p. 316). Segundo Kotler et al. (1993), marketing territorial tem como objetivo desenvolver uma comunidade que satisfaça diversos grupos de utilizadores (visitantes, residentes e trabalhadores), empresas e mercado desses locais. Para isso, as cidades devem conquistar o apoio do governo, sociedade, líderes ou instituições se tornando receptivas a novos negócios, 
desafios ou investidores que possam ser atraídos a contribuir em uma estratégia diferenciada do local (ARAGONEZ; ALVES, 2013). Cidrais (2001) reforça essa ideia conceituando o marketing territorial como responsável por potencializar a competitividade entre empresas proporcionando oportunidades de desenvolvimento territorial. Esse desenvolvimento possibilita que o grupo de utilizadores (população, entidades e governos) tenham acesso a um novo conceito de qualidade de vida, mobilidade urbana e desenvolvimento econômico.

A Figura 1 apresenta a interação entre os conceitos de marketing territorial e inteligência de mercado.

Figura 1 - Vantagem da utilização do Marketing territorial com IM/IC

Características do Marketing Territorial (MT)
- Potencializar competividade empresarial;
- Construção de redes colaborativa entre
cidades;
- Atendimento da demanda do grupo de
- utilizadores;
- Desenvolvimento de parcerias
- Atratividade para investidores;
- Desenvolvimento Territorial;
- Desenvolvimento econômico;

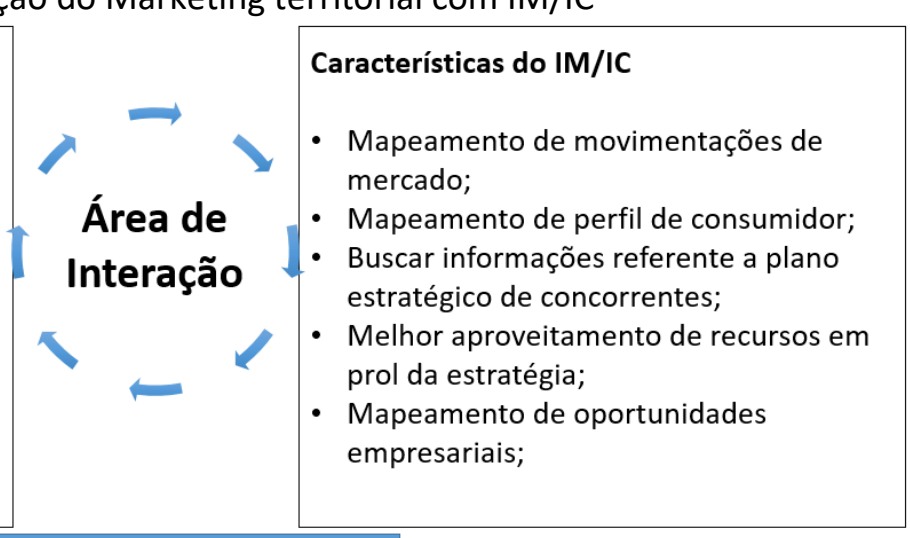

Oportunidades geradas com a utilização do Marketing Territorial alinhado com IM/IC

- Melhor aproveitamento de recursos;

- Aceleração do processo de inovação tecnológica;

- Fornecimento de produtos que melhorem a qualidade de vida;

- Criação de ponte entre negócios inovadores e investidores;

- Fomentar um ecossistema que influencia na mobilidade, acessibilidade e informação;

Fonte: modelo adaptado pelo autor referenciado aos conceitos de Castells (2005), Weng (2020), Kotler et al. (1993).

Ao correlacionar o marketing territorial com análises estratégicas de inteligência competitiva (IC) ou inteligência de mercado (IM), podemos entender que as oportunidades desenvolvidas e aprimoradas pelo marketing territorial poderão ser potencializadas pela utilização da inteligência de mercado ou inteligência competitiva, visto que suas principais características são mapear e interpretar pequenas movimentações ou estímulos do ambiente analisado. Em seu estudo, Aragonez e Alves 
(2013), apresenta a ideia defendida por Porter (1990) no livro "The competitive advantage of nations", onde afirma que nenhum país é competitivo em todas as áreas de negócios, porém é necessário que consigam moldar seus ambientes empresariais no qual suas empresas competem, com o objetivo de promover ou impedir a criação de vantagem competitiva. Essa ideia apoia-se em quatro pilares; condições de fatores (são criados e não herdados), condição da procura (a exigência dos consumidores), a relação entre as indústrias (o trabalho em rede), e a rivalidade, estrutura e estratégia das empresas (competição aumenta a produção e o grau de inovação) (ARAGONEZ; ALVES, 2013).

\section{PERCURSO METODOLÓGICO}

Para alcançar o objetivo desse estudo, será feita uma pesquisa utilizando por base o estudo realizado por Lazaroiu e Roscia (2012), onde identificou-se que a relação entre cidades inteligentes e as decisões humanas são interconectadas impossibilitando a diferenciação entre indicadores objetivos e indicadores subjetivos, segregando cidades a serem estudadas de acordo com o tamanho populacional e desenvolvimento econômico.

Conduziremos uma pesquisa comparativa entre iniciativas empreendedoras civis empresariais e governamentais. Utilizando ferramentas de entrevistas buscando entender como prefeitura e empreendedores utilizam técnicas de IM para monitorar oportunidades mercadológicas junto com dados fornecidos pelo Instituto Brasileiro de Geografia e Estatística (IBGE), índice de Potencial de Consumo (IPC Maps), Instituto de Pesquisa Econômica Aplicada (IPEA), Serviço Brasileiro de Apoio às Micro e Pequenas Empresas (SEBRAE), visando diferenciar a performance de cidades de acordo com a aplicação desses processos.

Este projeto de pesquisa será desenvolvido de acordo com o cronograma apresentado na Figura 2. 
Figura 2 - Cronograma de pesquisa

\begin{tabular}{|c|c|c|c|c|c|c|c|c|}
\hline & & & & & $\begin{array}{l}\text { Concluíd } \\
\text { Em anda } \\
\text { Não inici }\end{array}$ & $\begin{array}{l}\text { ento } \\
\text { lo }\end{array}$ & & \\
\hline Descrição do marco & Progresso & Início & № de dias & Q3'20 & Q4'20 & Q1'21 & Q2'21 & Q3'21 \\
\hline Desenvolvimento do Projeto de pesquisa & $100 \%$ & 01/06/2020 31/12/2020 & 213 & & & & & \\
\hline Revisão bibliográfica & $80 \%$ & $01 / 01 / 2021 \quad 26 / 02 / 2021$ & 56 & & & & & \\
\hline Elaboração dos instrumentos de coleta de dados & $10 \%$ & $27 / 02 / 202101 / 03 / 2021$ & 2 & & & & & \\
\hline Seleção da amostra & $0 \%$ & 02/03/2021 10/03/2021 & 8 & & & & & \\
\hline Coleta e análise de dados coletados & $0 \%$ & $11 / 03 / 2021 \quad 20 / 03 / 2021$ & 9 & & & & & \\
\hline Avaliação dos resultados & $0 \%$ & $21 / 03 / 202131 / 03 / 2021$ & 10 & & & & & \\
\hline Revisão & $0 \%$ & 01/04/2021 30/04/2021 & 29 & & & & & \\
\hline Qualificação & $0 \%$ & 01/05/2021 30/05/2021 & 29 & & & & & \\
\hline Defesa & $0 \%$ & $31 / 05 / 202103 / 06 / 2021$ & 3 & & & & & \\
\hline
\end{tabular}

Fonte: modelo desenvolvido pelo autor referente ao planejamento das atividades para a pesquisa acadêmica.

\section{CONSIDERAÇÕES PARCIAIS}

É possível entender que a complexidade do ecossistema de uma cidade inteligente proporciona muitas oportunidades para uma sociedade empreendedora, contudo conforme defendido por Perng (2019), sem a participação de agências governamentais ou organizações privadas por meio de hackathons ou similares eventos o progresso em cidades empreendedoras ou smart urbanas essa oportunidade estará comprometida. Contudo, independente dessas iniciativas as necessidades dos novos consumidores não param. Conforme defendido por César (2016), toda sociedade de consumidores necessita de rotas alternativas para manter suas atividades em funcionamento, se há escassez matérias - primas e piores condições de vidas, faz - se necessário a inovação.

Um ponto de atenção que essa pesquisa apresenta é referente a complexidade para um governo gerenciar todos os indicadores de sustentabilidade de modo que propiciem o desenvolvimento adequado do empreendedorismo, pois, os resultados econômicos de uma cidade inteligente será o resultado de um equilibro entre os eixos de desenvolvimento. 
Diante disso, esta pesquisa poderá contribuir para que seja possível identificar modelos ou maneiras onde a inteligência de mercado poderá auxiliar governos e empresas de forma conjunta potencializando o aproveitamento de ações de planejamento urbano alinhado ao planejamento estratégico empresaria gerando maiores benefícios a sociedade refletindo no desenvolvimento territorial e empreendedor de uma cidade inteligente.

\section{REFERÊNCIAS}

ARAGONEZ, Teresa; ALVES, Gonçalo Caetano. Marketing Territorial: O futuro das cidades sustentáveis e de sucesso. Tourism \& Management Studies, v. 1, p. 316-329, 2013.

CARVALHO, Luís; MAIA, Catarina. Empreendedores cívicos e Smart Cities: práticas, motivações e geografias da inovação. GOT. Revista de Geografia e Ordenamento do Território, Porto, n. 10, p. 95-112, 2016.

CASTELLS, Manuel. A sociedade em rede. Revista e ampliada. São Paulo: Paz e Terra, 2005.

CÉSAR, Vivian Aparecida Blaso Souza Soares. Cidades inteligentes: polissemias urbanas e pensamento complexo. 2016. 179 f. Tese (Doutorado em Ciências Sociais) - Programa de Estudos Pós-Graduados em Ciências Sociais, Pontifícia Universidade Católica de São Paulo, São Paulo, 2016.

CIDRAIS, Álvaro. O marketing territorial aplicado às cidades médias portuguesas: os casos de Évora e Portalegre. Biblio 3w: revista bibliográfica de geografía y ciencias sociales, Barcelona, v. 6, 2001.

DALMARCO, Denise. Gestão da informação e inteligência de mercado. São Paulo: Editora Senac, 2020.

DE JONG, Martin et al. Sustainable-smart-resilient-low carbon-eco-knowledge cities; making sense of a multitude of concepts promoting sustainable urbanization. Journal of Cleaner production, v. 109, p. 25-38, 2015.

FERNANDES, Ricardo Jorge Lopes; FERNANDES, Rui Jorge Gama. As Cidades e Territórios do Conhecimento na Óptica do Desenvolvimento e do Marketing Territorial. In: COLÓQUIO DA ASSOCIAÇÃO PORTUGUESA DE DESENVOLVIMENTO REGIONAL 
(APDR), 5., 2006, Lisboa. Actas do [...]. Lisboa: APDR e Escola Superior de Tecnologia de Viseu, 2006. Tema: "Recursos, Ordenamento, Desenvolvimento".

GIFFINGER, Rudolf et al. City-ranking of European medium-sized cities. In: [IFHP WORLD CONGRESS (“Future of Cities"), 51., 2007, Copenhagen. Vienna: Vienna University of Technology], 2007.

KITCHIN, Rob. The real-time city? Big data and smart urbanism. GeoJournal, v. 79, n. 1, p. 1-14, 2014.

KOTLER, Philip et al. Administração de Marketing. 4. ed. São Paulo: Editora Atlas, 1993.

KOURTIT, Karima; NIJKAMP, Peter; STOUGH, Roger (ed.). Quality and Inequality in Urban and Regional Systems. REGION, v. 5, n. 4, 2019.

LAZAROIU, George Cristian; ROSCIA, Mariacristina. Definition methodology for the smart cities model. Energy, v. 47, n. 1, p. 326-332, 2012.

MARTINET, Alain-Charles. Strategic planning, strategic management, strategic foresight: The seminal work of $\mathrm{H}$. Igor Ansoff. Technological Forecasting and Social Change, v. 77, n. 9, p. 1485-1487, 2010.

PERNG, Sung-Yueh. Promises, practices and problems of collaborative infrastructuring. In: COLLETA, Claudio et al. (ed.) Creating Smart Cities. Abingdon, Oxon; New York, NY: Routledge, 2019. Cap. 12, p.155-168. (Series Regions and Cities; v. 131)

SEBRAE. Data Sebrae Indicadores. Brasil, 2020. Disponível em: https://datasebraeindicadores.sebrae.com.br/resources/sites/data-sebrae/datasebrae.html. Acesso em: 26 ago. 2020.

SHAW, Michael J. et al. Knowledge management and data mining for marketing. Decision support systems, v. 31, n. 1, p. 127-137, 2001.

TARAPANOFF, Kira. Inteligência, informação e conhecimento em corporações. Brasília: Instituto Brasileiro de Informação em Ciência e Tecnologia (IBICT), 2006.

TEIXEIRA, Daniela Ramos. Tecnologias aplicadas à inteligência empresarial e inteligência competitiva no brasil? Revista Inteligência Competitiva, v. 1, n. 2, p. 239243, 2011.

TROMBIN, Vinícius Gustavo. O uso de cenários em marketing. 2014. Tese (Doutorado em Administração) - Faculdade de Economia, Administração e Contabilidade, 
Universidade de São Paulo, São Paulo, 2014. Doi: 10.11606/T.12.2014.tde-24062014152114.

URBAN SYSTEM. Ranking Connected Smart Cities. Brasil, 2020. Disponível em: https://www.urbansystems.com.br/rankingconnectedsmartcities. Acesso em: 26 ago. 2020.

WENG, Wilson. Effect of Internet of Things on Business Strategy: The Mediating Role of Marketing Intelligence Capability. EasyChair Preprint n. 2729, 2020.

\section{Declaração de Contribuição dos Autores}

André Luiz Gomes: Conceptualização - Curadoria de Dados - Análise Formal Investigação - Metodologia - Administração do Projeto - Validação - Visualização Escrita.

Taiane Ritta Coelho: Conceptualização - Administração do Projeto - Supervisão Validação - Visualização - Escrita. 